\title{
Spontaneous fractional Chern insulators in transition metal dichalcogenide moiré superlattices
}

\author{
Heqiu Li $\odot,{ }^{1}$ Umesh Kumar $\odot,{ }^{2}$ Kai Sun, ${ }^{1}$ and Shi-Zeng Lin $\odot^{3}$ \\ ${ }^{1}$ Department of Physics, University of Michigan, Ann Arbor, Michigan 48109, USA \\ ${ }^{2}$ Theoretical Division, T-4, Los Alamos National Laboratory, Los Alamos, New Mexico 87545, USA \\ ${ }^{3}$ Theoretical Division, T-4 and CNLS, Los Alamos National Laboratory, Los Alamos, New Mexico 87545, USA
}

(Received 4 January 2021; revised 15 June 2021; accepted 24 August 2021; published 22 September 2021)

\begin{abstract}
The Moiré superlattice realized in two-dimensional heterostructures offers an exciting platform to access strongly correlated electronic states. In this work, we study transition metal dichalcogenides (TMD) Moiré superlattices with time-reversal symmetry and nontrivial spin/valley-Chern numbers. Utilizing realistic material parameters and the method of exact diagonalization, we find that at a certain twisting angle and fractional filling, gapped fractional topological states, i.e., fractional Chern insulators, are naturally stabilized by simply introducing the Coulomb repulsion. In contrast to fractional quantum Hall systems, where the time-reversal symmetry has to be broken explicitly, these fractional states break the time-reversal symmetry spontaneously. We show that the Chern number contrasting in the opposite valleys imposes a strong constraint on the nature of fractional Chern insulator and the associated low-energy excitations.
\end{abstract}

DOI: 10.1103/PhysRevResearch.3.L032070

Introduction. When two layers of two-dimensional materials are placed on top of each other with slight misalignment it creates a superlattice with periodicity much larger than the atomic lattice parameter. Because of the large lattice periodicity, one can fill or empty the entire band by electrode gating. This Moiré superlattice provides a tunable platform to control the electronic band structure $[1,2]$, and therefore enables access to a plethora of interesting quantum states. Because the bandwidth in these systems can be tuned to be extremely narrow [2], these Moiré superlattices open up a new pathway to stabilize various strongly correlated phases, such as superconductivity and correlated insulators [3-27]. Furthermore, such electronic band structure can also be topologically nontrivial, e.g., with a nonzero integer Chern number [28-32]. Combined with their strong coupling nature, such Moiré superlattices offer a promising route to realize the longsought fractionalized topological order [33-39].

Recently, gapped electronic states at various fractional fillings (e.g., 1/3) were observed in transition metal dichalcogenide (TMD) Moiré superlattices, e.g., $\mathrm{WSe}_{2} / \mathrm{WS}_{2}$ [18,40-43]. In general, gapped electronic states at fractional filling may have two origins: (i) charge order that spontaneously breaks the translational symmetry and (ii) fractional topological order, e.g., fractional Chern insulators (FCI) [44-51]. In these TMD Moiré superlattices, the observed gapped states were interpreted as Wigner crystals of electrons because the underlying single-particle bands are topologically trivial [52].

Published by the American Physical Society under the terms of the Creative Commons Attribution 4.0 International license. Further distribution of this work must maintain attribution to the author(s) and the published article's title, journal citation, and DOI.
Encouraged by such exciting experimental progress, here we explore the feasibility of the second category in TMD Moiré superlattices. In particular, we focus on systems like $\mathrm{MoTe}_{2}$, which may host topologically nontrivial bands with nonzero spin/valley-Chern numbers [53]. In contrast to a partially filled Chern band [44-51], because these systems preserve the time-reversal symmetry, two types of fractional states are, in principle, allowed (i) time-reversal invariant fractional topological insulators [54] and (ii) FCIs via spontaneously breaking the time-reversal symmetry. The key focus of this study is whether Coulomb repulsion could stabilize some of these fractional states in TMD Moiré superlattices.

In this work, we show that by simply increasing the Coulomb interaction strength in such TMD Moiré superlattices, the system undergoes a quantum phase transition that spontaneously breaks the time-reversal symmetry by polarizing electrons into one of the two valleys. Further increase of Coulomb interaction will trigger a second quantum phase transition and stabilize a FCI at a fractional filling. For excitations, our numerical studies observe both (intravalley) fractional excitations from the fractional topological order and (intervalley) valley-wave excitations from the spontaneous symmetry breaking. We argue that the symmetry breaking state and low-energy excitations are constrained by the valley contrasting Chern number in the TMD Moiré superlattice.

Model. We consider twisted homobilayer TMD materials. For each single layer, the low-energy electronic states reside at the valence band maxima at $\pm \boldsymbol{K}$ valleys. Contrary to bilayer graphene systems where the valley and spin degrees of freedom are both present, in TMD each valley in the top valence band has fixed spin orientation due to strong spinorbit coupling and the broken inversion symmetry [55]. With a small twist angle $\theta$ between two layers, the $+\boldsymbol{K}$ valley for the top and bottom layers are shifted to $\boldsymbol{K}_{t}$ and $\boldsymbol{K}_{b}$ in the Moiré Brillouin zone (MBZ), respectively, [Fig. 1(b)]. For 
(a)

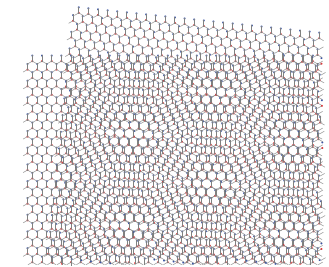

(c)

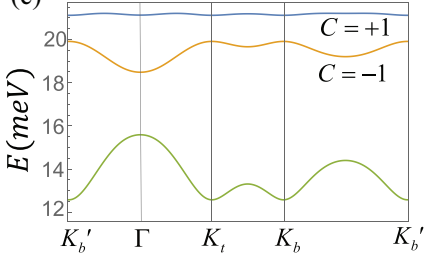

(b)

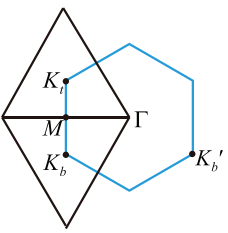

(d)

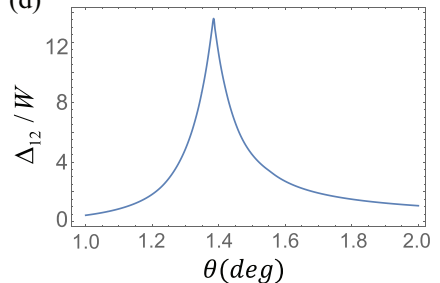

FIG. 1. (a) Schematic view of the Moiré superlattice. (b) We choose the Moiré Brillouin zone (MBZ) to be the rhombus and the origin in momentum space is chosen at $M$. (c) Moiré band structure at $\theta=1.38^{\circ}$. The top Moiré band is nearly flat with Chern number \pm 1 . (d) The gap ratio $\frac{\Delta_{12}}{W}=\frac{\min \left[E_{1}(k)\right]-\max \left[E_{2}(k)\right]}{\max \left[E_{1}(k)\right]-\min \left[E_{1}(k)\right]}$ as a function of twisted angle $\theta$, where $E_{1}(\boldsymbol{k})\left[E_{2}(\boldsymbol{k})\right]$ is the energy of the first (second) topmost Moiré band.

convenience we choose the rhombus-shaped MBZ and set the point $\boldsymbol{M}=\left(\boldsymbol{K}_{t}+\boldsymbol{K}_{b}\right) / 2$ as the origin. We employ the continuum model [2] in which the Moiré Hamiltonian for the $+\boldsymbol{K}$ valley is

$$
H_{+}(\boldsymbol{k}, \boldsymbol{r})=\left(\begin{array}{cc}
-\frac{\hbar^{2}\left(\boldsymbol{k}-\boldsymbol{K}_{b}\right)^{2}}{2 m^{*}}+\Delta_{\mathfrak{b}}(\boldsymbol{r}) & \Delta_{T}(\boldsymbol{r}) \\
\Delta_{T}^{\dagger}(\boldsymbol{r}) & -\frac{\hbar^{2}\left(\boldsymbol{k}-\boldsymbol{K}_{t}\right)^{2}}{2 m^{*}}+\Delta_{\mathrm{t}}(\boldsymbol{r})
\end{array}\right) .
$$

Here $m^{*}$ is the effective mass. The form of the Moiré potential, $\Delta_{b, t, T}$, is dictated by the $D_{3}$ crystalline symmetry and a combination of $C_{2 z}$ rotation followed by switching the two layers, and can be parameterized by [53]

$$
\begin{aligned}
\Delta_{T}(\boldsymbol{r}) & =w\left(1+e^{-i \boldsymbol{G}_{2} \cdot \boldsymbol{r}}+e^{-i \boldsymbol{G}_{3} \cdot \boldsymbol{r}}\right), \\
\Delta_{l}(\boldsymbol{r}) & =2 w_{z} \sum_{j=1,3,5} \cos \left(\boldsymbol{G}_{j} \cdot \boldsymbol{r}+l \psi\right),
\end{aligned}
$$

where $l \in\{b, t\}=\{+1,-1\}$ and $\boldsymbol{G}_{j}$ is the Moiré reciprocal lattice vectors with length $\left|\boldsymbol{G}_{j}\right|=\frac{4 \pi}{\sqrt{3} a_{M}}$ and polar angle $\frac{\pi(j-1)}{3}$. Here $a_{M}=a_{0} / \theta$ is the Moiré lattice constant for a small twisted angle $\theta$ and $a_{0}$ is the lattice parameter of TMD. The Hamiltonian for the valley $-\boldsymbol{K}$ can be obtained by the time-reversal symmetry $H_{-}(\boldsymbol{k}, \boldsymbol{r})=H_{+}(-\boldsymbol{k}, \boldsymbol{r})^{*}$. To be specific, we focus on the twisted $\mathrm{MoTe}_{2}$ homobilayer with typical parameters $\left(\hbar^{2} / 2 m^{*} a_{0}^{2}, w_{z}, w, \psi\right)=$ (495 meV, $8 \mathrm{meV},-8.5 \mathrm{meV},-89.6^{\circ}$ ) [53].

The top valence band of a TMD single layer splits into multiple Moiré bands due to the Moiré potential. As shown in Figs. 1(c) and 1(d), when the twist angle is close to $\theta_{0}=$ $1.38^{\circ}$, the top Moiré band becomes nearly flat. The flatness of a band can be characterized by a ratio of the gap between the nearest bands to its bandwidth. For the top Moiré band, the ratio can be as large as 13 . When $\theta<3.1^{\circ}$, The top Moiré band is topologically characterized by a valley/spin Chern number $C= \pm 1$ due to the skyrmion lattice pseudospin textures of the Moiré potential [53]. The Chern number for the opposite valley/spin is opposite as required by time-reversal symmetry. Thus, at the single-particle level, such TMD homobilayer realizes a quantum valley/spin Hall insulator.

We then introduce the screened Coulomb interaction and project it to the nearly flat top Moiré band [56]

$$
\begin{aligned}
H_{\mathrm{int}}= & \frac{1}{2 A} \sum_{\boldsymbol{q}}: \rho(\boldsymbol{q}) V(\boldsymbol{q}) \rho(-\boldsymbol{q}): \\
= & \sum_{\boldsymbol{k}, \boldsymbol{k}^{\prime}, \boldsymbol{q}, \tau, \tau^{\prime}} \frac{U}{2 N_{\mathrm{cell}}} v(\boldsymbol{q}) \lambda_{\tau, \boldsymbol{q}}(\boldsymbol{k}) \lambda_{\tau^{\prime}, \boldsymbol{q}}\left(\boldsymbol{k}^{\prime}\right)^{*}, \\
& C_{\tau}^{\dagger}(\boldsymbol{k}) C_{\tau^{\prime}}^{\dagger}\left(\boldsymbol{k}^{\prime}+\boldsymbol{q}\right) C_{\tau^{\prime}}\left(\boldsymbol{k}^{\prime}\right) C_{\tau}(\boldsymbol{k}+\boldsymbol{q}),
\end{aligned}
$$

where $\tau= \pm$ is the valley index and $\lambda_{\tau, \boldsymbol{q}}(\boldsymbol{k})=\left\langle u_{\tau, \boldsymbol{k}} \mid u_{\tau, \boldsymbol{k}+\boldsymbol{q}}\right\rangle$ is the form factor originated from the projection. Here $v(\boldsymbol{q})=$ $4 \pi \tanh (q d) / \sqrt{3} q a_{M}$ is the dimensionless screened Coulomb potential with $d$ the separation between the electrode and Moiré superlattice, which is set to $d=2 a_{M}$ in the calculations. $A$ is system area and $N_{\text {cell }}$ is the number of the unit cells in the calculations. The coefficient of $v(\boldsymbol{q})$ is chosen to make $U$ equal to the bare Coulomb potential between two particles separated by $a_{M} . C_{\tau}(\boldsymbol{k})$ is the annihilation operator for the single-particle state $\left|u_{\tau, k}\right\rangle$. We neglect the weak intervalley impurity scattering process associated with a large momentum transfer, and therefore the Hamiltonian also has a valley $U(1)_{v}$ symmetry. In this model, there are two competing symmetry breaking states: An intervalley coherent state that breaks the valley $U(1)_{v}$ symmetry and a valley/spin polarized state that breaks the time-reversal symmetry. At half filling of the topmost band (account for the valley degree of freedom), our Hartree-Fock analysis and exact-diagonalization results both suggest that a valley-polarized state is energetically favored, which spontaneously breaks the time-reversal symmetry and leads to an interaction-induced Chern insulator [57]. At fractional filling, in principle, two types of fractional topological states might emerge, a fractional Chern insulator or a fractional topological insulator [54,58], depending on whether the time-reversal symmetry is spontaneously broken or preserved [59], and our exact diagonalization below shows that the FCI is favored and stabilized in our system.

Valley polarized FCI. We define the filling factor $v=$ $2 \rho_{e} / \rho_{s}$, where $\rho_{e}$ is the electron density occupying the top Moiré band and $\rho_{s}$ is the electron density for the full filling of the two-fold degenerate top Moiré band. The factor of 2 accounts for the valley degree of freedom. Using exact diagonalization, at $v=1 / 3$ we observe numerical evidence of spontaneous valley polarization and FCI in the strong interaction limit, as shown in Fig. 2(a). For eight electrons in $4 \times 6$ unit cells $(4 \times 6 \times 2$ single-particle states including both valleys), the ground states are fully valley polarized with three nearly degenerate ground states for each valley polarization, separated from the excited states by an energy gap of the order of $2 \mathrm{~K}$. We calculated the many-body Chern number of each ground state [48], and the topological index is found to be $1 / 3$, characterizing a $1 / 3$ FCI phase. This conclusion is further supported by the total momentum for each ground state, which obeys the generalized Pauli exclusion rule [60].

The occupation number $n\left(k_{1}, k_{2}\right)$ of single-particle states for each of the three many-body ground states are plotted in Fig. 2(b). $n\left(k_{1}, k_{2}\right)$ is uniformly distributed for different 

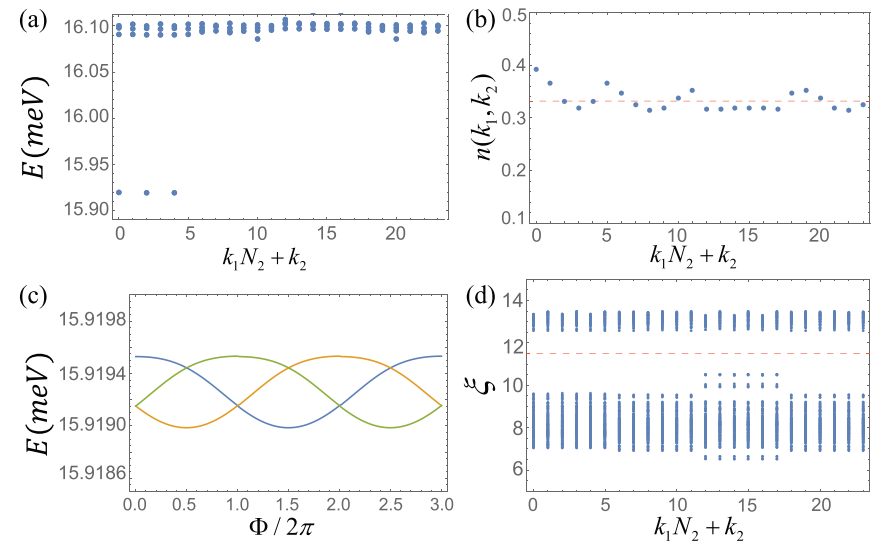

FIG. 2. Numerical diagonalization results for eight particles in $4 \times 6$ Moiré lattice. We choose $\theta=1.38^{\circ}$ and $U=1.38 \mathrm{meV}$. The bandwidth at this twist angle is $W=0.083 \mathrm{meV}$. Here $N=N_{1} \times$ $N_{2} / 3$ is the number of particles. (a) Energy spectrum with three nearly degenerate ground states in each valley. (b) The occupation number of single particle states $n\left(k_{1}, k_{2}\right)$ for each of the three manybody ground states. The nearly uniform distribution of $n\left(k_{1}, k_{2}\right)$ suggests the ground state is an incompressible liquid. (c) Under flux insertion along $k_{2}$ direction, the ground states evolve into each other. (d) Particle entanglement spectrum (PES) for the separation of $N_{A}=4$ particles.

single-particle states, consistent with the fact that the ground state is an incompressible liquid. The spectrum evolution under flux insertion along the $k_{2}$ direction is shown in Fig. 2(c). The excitation gap is maintained throughout the flux insertion process.

The topological nature of the ground states are further confirmed by our calculation of the particle entanglement spectrum (PES) [60]. To compute PES, we divide the $N$ particles into two collections of $N_{A}$ and $N_{B}=N-N_{A}$ particles and trace out $N_{B}$ particles to get the reduced density matrix $\rho_{A}$. The PES levels $\xi$ are obtained from the logarithm of eigenvalues of $\rho_{A}$, and are labeled by the total momentum of the remaining $N_{A}$ particles, as shown in Fig. 2(d). There is a clear entanglement gap with 2730 levels below the gap for $N_{A}=4$, consistent with the counting of quasihole excitation in the $v=1 / 3$ FCI [60].

To examine the finite-size effect, we study the scaling of the many-body gap $\Delta$ with various system sizes. For a genuine FCI, $\Delta$ remains finite in the thermodynamic limit when both $N_{1}$ and $N_{2}$ approach infinity. However, $\Delta$ should vanish if only one of $N_{1}$ or $N_{2}$ approaches infinity because this limit is a one-dimensional system which should not support FCI [47]. This is confirmed in Fig. 3(a), which shows $\Delta$ decreases when $N_{1}$ is fixed at 3 and $N_{2}$ increases from 4 to 8 , but $\Delta$ increases when the system size changes from $3 \times 8$ to $4 \times 6$.

We then map out the phase diagram at $v=1 / 3$ filling as a function of the interaction strength $U$, which can be controlled by distance between the electrodes and the Moiré superlattice in experiments and the dielectric constant $\epsilon$. The results are shown in Fig. 3(b). We find a valley nonpolarized Fermi liquid at a small $1 / \epsilon$ corresponding to a small $U$, a Fermi liquid with valley polarization at an intermediate interaction, and the FCI phase with valley polarization at strong interaction. (a)

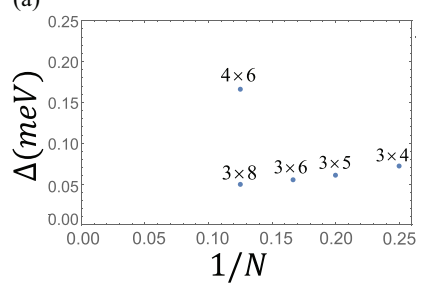

(b)

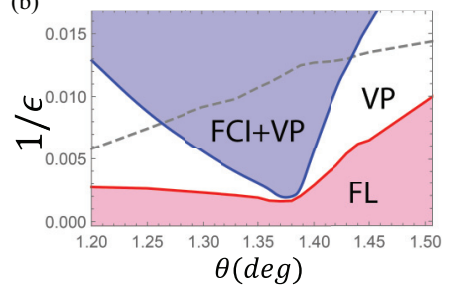

FIG. 3. (a) The many-body gap $\Delta$ for various system sizes at $v=$ $1 / 3$ filling. The interaction strength is fixed to be $U=1.38 \mathrm{meV}$. The increase of $\Delta$ in $4 \times 6$ system suggests the gap persists in the twodimensional thermodynamic limit. (b) The phase diagram for Fermi liquid (FL), FL with valley polarization (VP) and fractional Chern insulator (FCI) at different interaction strength $U(\epsilon)=\frac{e^{2}}{4 \pi \epsilon \epsilon_{0} a_{M}}$ and twisted angle $\theta$. The dashed line corresponds to $U(\epsilon)=\Delta_{12}$, above which the interaction starts to mix different bands and the singleband approximation breaks down.

Depending on the twisted angle $\theta$, which controls the bandwidth, the valley nonpolarized Fermi liquid can transit directly to FCI with valley polarization or through an intermediate Fermi liquid with valley polarization. The phase transition between the valley-polarized Fermi liquid and FCI can be described by Ginzburg-Landau theory with a Chern-Simons term [57]. The direct transition occurs near $\theta=1.38^{\circ}$ where the single-particle Moiré band has the largest gap to bandwidth ratio [see Fig. 1(d)]. This is consistent with the quantum Hall systems with flat Landau levels, where the interaction stabilizes simultaneously the fractional quantum Hall state with spin polarization. Note that the FCI can be stabilized in a relatively broader region of the twisted angle here compared to that in the magic angle twisted bilayer graphene [33-39], and the region of angle for FCI increases with interaction. For interaction above the dashed line in Fig. 3(b), our single-band approximation used in the numerical calculations breaks down and it requires taking other nearby bands into account.

Excitations. Here we study the charge neutral excitations above the FCI ground states. As a consequence of the spontaneous valley polarization, we consider the valley waves' excitation $\left|\Psi_{v}(\boldsymbol{q})\right\rangle=\sum_{\boldsymbol{k}} z_{k} C_{+}^{\dagger}(\boldsymbol{k}+\boldsymbol{q}) C_{-}(\boldsymbol{k})\left|\Psi_{-}\right\rangle$, where $\left|\Psi_{-}\right\rangle$is the FCI ground state with the $\tau=-$ valley fully occupied and $z_{k}$ is a variational parameter. The presence of the form factor in Eq. (3) breaks the valley pseudospin $\mathrm{SU}(2)$ rotation symmetry down to the valley $U(1)_{v}$ symmetry. As a result, the valley wave excitation are gapped as shown in Fig. 4, which can be fitted by $E_{w}(\boldsymbol{q})=J q^{2}+A$. The valley wave disperses weakly in momentum and thus is well localized in real space.

The lowest intravalley many-body excitation has lower energy than the valley wave excitation for the parameters we used, i.e., the energy difference between the lowest fully polarized excited state and the FCI state is $E_{m b}=0.167 \mathrm{meV}<$ $E_{w}$, see Fig. 4. Nevertheless, the valley wave excitation remains a stable excitation because the decay of the valley wave to the intravalley many-body excitations are forbidden. Intravalley many-body excitations have the valley quantum number 0 , while the valley wave has the valley quantum number 2 . 


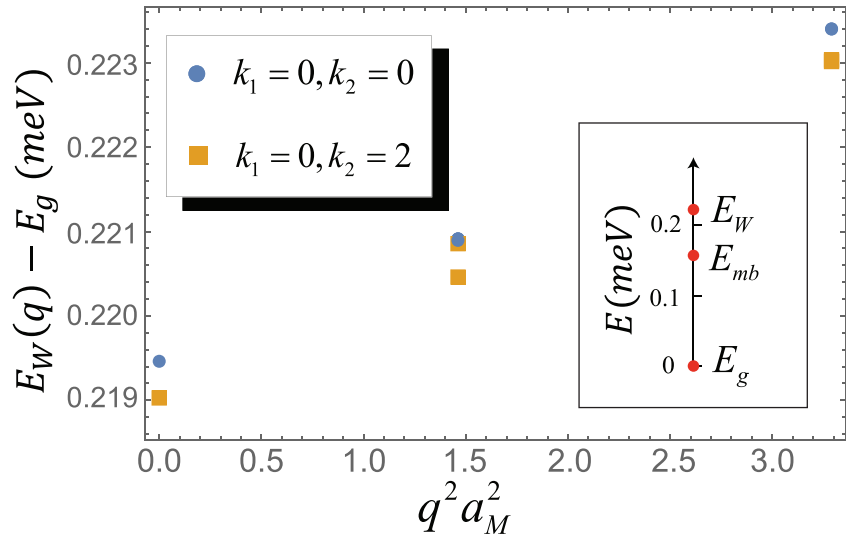

FIG. 4. Dispersion of valley wave excitation $E_{w}(\boldsymbol{q})$ for eight particles in $4 \times 6$ lattice. Excitation above the ground state with total momentum $k_{1}=0, k_{2}=0\left(k_{1}=0, k_{2}=2\right)$ are labeled by the squares and circles, respectively. The slight energy difference for these two ground states is caused by finite-size effect. The inset compares the energy of the lowest valley wave excitation $E_{w}$, the lowest intravalley many-body excitation $E_{m b}$, and the ground-state energy $E_{g}$.

In quantum Hall ferromagnets, a pair of skyrmions has lower energy than the particle-hole bound state [61]. The system size limitation in the exact diagonalization does not allow us to study the valley skyrmion excitation in our numerical calculations. Here we use an effective Hamiltonian density for valley pseudospin $\mathbf{n}(r)$ [61]

$$
H_{n}(r)=\frac{J}{2}(\nabla \mathbf{n})^{2}-\frac{A}{2} n_{z}^{2}+\frac{1}{2} \int d r^{\prime 2} V\left(r-r^{\prime}\right) \rho_{s}(r) \rho_{s}\left(r^{\prime}\right),
$$

where $J$ and $A$ are given by the valley wave spectrum. The presence of the valley pseudospin anisotropy can be traced back to the opposite Chern number for the opposite valley. One cannot rotate $\mathbf{n}$ from one valley to opposite valley adiabatically without closing the energy gap, which implies the existence of anisotropy for $\mathbf{n}$. The last term accounts for the Coulomb interaction $V\left(r-r^{\prime}\right)$ because a skyrmion is dressed with charge distribution $\rho_{s}(r)=\epsilon^{i j} \epsilon_{a b c} n^{a} \partial_{i} n^{b} \partial_{j} n^{c} / 8 \pi$, where $\epsilon_{a b c}\left(\epsilon^{i j}\right)$ is the Levi-Civita tensor with $i, j$ being the space index and $a, b, c$ being the spin index. The skyrmion topological charge $Q_{s}=\int d r^{2} \rho_{s}(r)$ is quantized to an integer number. The easy axis anisotropy favors skyrmions with a small radius while the Coulomb repulsion favors skyrmions with a large radius. Their competition determines the skyrmion size [62].

FCI at $v=2 / 5$. In twisted graphene Moiré superlattices, the Halperin (332) state is stabilized at $v=2 / 5$ due to the remaining $S U(2)$ spin rotation symmetry in the valley polarized state [36]. In our TMD Moiré superlattices, the spin rotation symmetry is absent because of the spin-valley locking. The Chern number contrasting valley degree of freedom disfavors the (332) state. To demonstrate this explicitly, we calculate the energy spectrum, spectrum flow under flux insertion, and entanglement spectrum at $v=2 / 5$, and the results are displayed
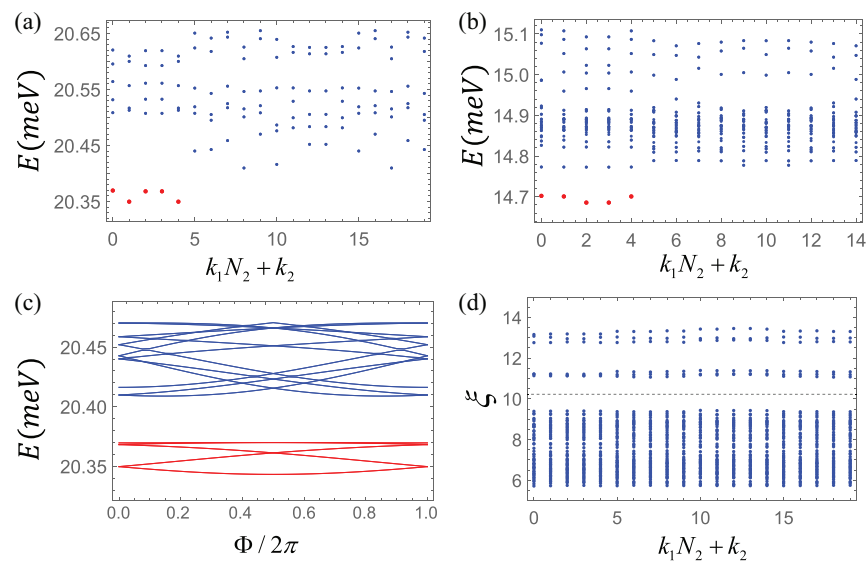

FIG. 5. FCI at $v=2 / 5$. (a) The energy spectrum of eight particles in $4 \times 5$ system, and (b) six particles in $3 \times 5$ system. (c) The flux insertion for the system in (a), where the five ground states are marked in red (some of them are on top of each other). A finite gap remains during flux insertion. (d) The particle entanglement spectrum for (a) with $N_{A}=3$. There are $51 \times 20=1020$ states below the dashed line, consistent with quasihole counting.

in Fig. 5. The five-fold degenerate ground states are valley polarized, and are consistent with the $v=2 / 5$ FCI state. In the fractional quantum Hall, the $v=2 / 5$ state belongs to the second hierarchical Jain state, and similarly one can assign the $v=2 / 5$ FCI as a second hierarchical FCI. Our results highlight the importance of symmetry in dictating the ground state and contrast the difference between the graphene and TMD Moire superlattices.

Discussions. We show that TMD Moiré superlattices can host fractional topological states via spontaneously breaking the time-reversal symmetry, using realistic parameters of TMD Moiré superlattices. Comparing with graphene, the spin-valley locking in TMD materials breaks the SU(2) spin rotation symmetry and eliminates the spin-wave Goldstone modes, which could help stabilize the FCI states. The valley contrasting Chern number in TMD Moiré superlattices also dictates the symmetry breaking states, hence the nature of fractionalized topological states, and also the low-energy excitations in the FCI. The gapped nature of these states can be detected by transport, optical measurements, and so on, and its topological nature can be accessed by the Hall conductivity measurement. Due to the strong analogy between FCI and chiral spin liquids, it is plausible that Moiré superlattices may also help realize/stabilize exotic spin liquid phases [63-65] by unitizing the valley/layer pseudospin or real spin degrees of freedom.

Acknowledgments This work done at LANL was carried out under the auspices of the U.S. DOE NNSA under Contract No. 89233218CNA000001 through the LDRD Program. S. Z. L. was also supported by the U.S. Department of Energy, Office of Science, Basic Energy Sciences, Materials Sciences and Engineering Division, Condensed Matter Theory Program. H.L. and K.S. acknowledge support through NSF Grant No. NSF-EFRI-1741618. 
[1] J. M. B. Lopes dos Santos, N. M. R. Peres, and A. H. Castro Neto, Graphene Bilayer with a Twist: Electronic Structure, Phys. Rev. Lett. 99, 256802 (2007).

[2] R. Bistritzer and A. H. MacDonald, Moiré bands in twisted double-layer graphene, Proc. Natl. Acad. Sci. USA 108, 12233 (2011).

[3] Y. Cao, V. Fatemi, A. Demir, S. Fang, S. L. Tomarken, J. Y. Luo, J. D. Sanchez-Yamagishi, K. Watanabe, T. Taniguchi, E. Kaxiras, R. C. Ashoori, and P. Jarillo-Herrero, Correlated insulator behaviour at half-filling in magic-angle graphene superlattices, Nature (London) 556, 80 (2018).

[4] Y. Cao, V. Fatemi, S. Fang, K. Watanabe, T. Taniguchi, E. Kaxiras, and P. Jarillo-Herrero, Unconventional superconductivity in magic-angle graphene superlattices, Nature (London) 556, 43 (2018).

[5] X. Lu, P. Stepanov, W. Yang, M. Xie, M. A. Aamir, I. Das, C. Urgell, K. Watanabe, T. Taniguchi, G. Zhang, A. Bachtold, A. H. MacDonald, and D. K. Efetov, Superconductors, orbital magnets and correlated states in magic-angle bilayer graphene, Nature (London) 574, 653 (2019).

[6] M. Yankowitz, S. Chen, H. Polshyn, Y. Zhang, K. Watanabe, T. Taniguchi, D. Graf, A. F. Young, and C. R. Dean, Tuning superconductivity in twisted bilayer graphene, Science $\mathbf{3 6 3}$, 1059 (2019).

[7] A. Kerelsky, L. J. McGilly, D. M. Kennes, L. Xian, M. Yankowitz, S. Chen, K. Watanabe, T. Taniguchi, J. Hone, C. Dean et al., Maximized electron interactions at the magic angle in twisted bilayer graphene, Nature (London) 572, 95 (2019).

[8] Y. Cao, D. Chowdhury, D. Rodan-Legrain, O. Rubies-Bigordà, K. Watanabe, T. Taniguchi, T. Senthil, and P. Jarillo-Herrero, Strange metal in magic-angle graphene with near planckian dissipation, Phys. Rev. Lett. 124, 076801 (2020).

[9] H. Polshyn, M. Yankowitz, S. Chen, Y. Zhang, K. Watanabe, T. Taniguchi, C. R. Dean, and A. F. Young, Large linear-intemperature resistivity in twisted bilayer graphene, Nat. Phys. 15, 1011 (2019).

[10] Y. Xie, B. Lian, B. Jäck, X. Liu, C.-L. Chiu, K. Watanabe, T. Taniguchi, B. A. Bernevig, and A. Yazdani, Spectroscopic signatures of many-body correlations in magic-angle twisted bilayer graphene, Nature (London) 572, 101 (2019).

[11] Y. Jiang, X. Lai, K. Watanabe, T. Taniguchi, K. Haule, J. Mao, and E. Y. Andrei, Charge order and broken rotational symmetry in magic-angle twisted bilayer graphene, Nature (London) 573, 91 (2019).

[12] Y. Choi, J. Kemmer, Y. Peng, A. Thomson, H. Arora, R. Polski, Y. Zhang, H. Ren, J. Alicea, G. Refael et al., Electronic correlations in twisted bilayer graphene near the magic angle, Nat. Phys. 15, 1174 (2019).

[13] U. Zondiner, A. Rozen, D. Rodan-Legrain, Y. Cao, R. Queiroz, T. Taniguchi, K. Watanabe, Y. Oreg, F. von Oppen, A. Stern, E. Berg, P. Jarillo-Herrero, and S. Ilani, Cascade of phase transitions and Dirac revivals in magic-angle graphene, Nature (London) 582, 203 (2020).

[14] D. Wong, K. P. Nuckolls, M. Oh, B. Lian, Y. Xie, S. Jeon, K. Watanabe, T. Taniguchi, B. A. Bernevig, and A. Yazdani, Cascade of electronic transitions in magic-angle twisted bilayer graphene, Nature (London) 582, 198 (2020).

[15] K. P. Nuckolls, M. Oh, D. Wong, B. Lian, K. Watanabe, T. Taniguchi, B. A. Bernevig, and A. Yazdani, Strongly correlated Chern insulators in magic-angle twisted bilayer graphene, Nature (London) 588, 610 (2020).

[16] M. He, Y. Li, J. Cai, Y. Liu, K. Watanabe, T. Taniguchi, X. $\mathrm{Xu}$, and $\mathrm{M}$. Yankowitz, Symmetry breaking in twisted double bilayer graphene, Nat. Phys. 17, 26 (2020).

[17] X. Liu, Z. Hao, E. Khalaf, J. Y. Lee, Y. Ronen, H. Yoo, D. Haei Najafabadi, K. Watanabe, T. Taniguchi, A. Vishwanath, and P. Kim, Tunable spin-polarized correlated states in twisted double bilayer graphene, Nature (London) 583, 221 (2020).

[18] E. C. Regan, D. Wang, C. Jin, M. I. Bakti Utama, B. Gao, X Wei, S. Zhao, W. Zhao, Z. Zhang, K. Yumigeta, M. Blei, J. D. Carlström, K. Watanabe, T. Taniguchi, S. Tongay, M. Crommie, A. Zettl, and F. Wang, Mott and generalized Wigner crystal states in WSe 2/WS 2 moiré superlattices, Nature (London) 579, 359 (2020).

[19] L. Wang, E.-M. Shih, A. Ghiotto, L. Xian, D. A. Rhodes, C. Tan, M. Claassen, D. M. Kennes, Y. Bai, B. Kim, K. Watanabe, T. Taniguchi, X. Zhu, J. Hone, A. Rubio, A. N. Pasupathy, and C. R. Dean, Correlated electronic phases in twisted bilayer transition metal dichalcogenides, Nat. Mater. 19, 861 (2020).

[20] M. Xie and A. H. MacDonald, Nature of the Correlated Insulator States in Twisted Bilayer Graphene, Phys. Rev. Lett. 124, 097601 (2020).

[21] F. Wu and S. Das Sarma, Collective Excitations of Quantum Anomalous Hall Ferromagnets in Twisted Bilayer Graphene, Phys. Rev. Lett. 124, 046403 (2020).

[22] Y. Su and S.-Z. Lin, Current-Induced Reversal of Anomalous Hall Conductance in Twisted Bilayer Graphene, Phys. Rev. Lett. 125, 226401 (2020).

[23] B. Padhi, C. Setty, and P. W. Phillips, Doped twisted bilayer graphene near magic angles: Proximity to wigner crystallization, not mott insulation, Nano Lett. 18, 6175 (2018).

[24] B. Padhi, R. Chitra, and P. W. Phillips, Generalized wigner crystallization in moiré materials, Phys. Rev. B 103, 125146 (2021).

[25] B. Padhi and P. W. Phillips, Pressure-induced metal-insulator transition in twisted bilayer graphene, Phys. Rev. B 99, 205141 (2019).

[26] N. Stefanidis and I. Sodemann, Excitonic laughlin states in ideal topological insulator flat bands and their possible presence in moiré superlattice materials, Phys. Rev. B 102, 035158 (2020).

[27] N. Bultinck, S. Chatterjee, and M. P. Zaletel, Mechanism for Anomalous Hall Ferromagnetism in Twisted Bilayer Graphene, Phys. Rev. Lett. 124, 166601 (2020).

[28] Y.-H. Zhang, D. Mao, Y. Cao, P. Jarillo-Herrero, and T. Senthil, Nearly flat chern bands in moiré superlattices, Phys. Rev. B 99, 075127 (2019).

[29] C. Repellin, Z. Dong, Y.-H. Zhang, and T. Senthil, Ferromagnetism in Narrow Bands of Moiré Superlattices, Phys. Rev. Lett. 124, 187601 (2020).

[30] A. L. Sharpe, E. J. Fox, A. W. Barnard, J. Finney, K. Watanabe, T. Taniguchi, M. A. Kastner, and D. GoldhaberGordon, Emergent ferromagnetism near three-quarters filling in twisted bilayer graphene, Science 365, 605 (2019).

[31] M. Serlin, C. L. Tschirhart, H. Polshyn, Y. Zhang, J. Zhu, K. Watanabe, T. Taniguchi, L. Balents, and A. F. Young, Intrinsic quantized anomalous hall effect in a moiré heterostructure, Science 367, 900 (2019).

[32] G. Chen, A. L. Sharpe, E. J. Fox, Y.-H. Zhang, S. Wang, L. Jiang, B. Lyu, H. Li, K. Watanabe, T. Taniguchi et al., 
Tunable correlated chern insulator and ferromagnetism in trilayer graphene/boron nitride moiré superlattice, arXiv:1905.06535.

[33] P. J. Ledwith, G. Tarnopolsky, E. Khalaf, and A. Vishwanath, Fractional chern insulator states in twisted bilayer graphene: An analytical approach, Phys. Rev. Research 2, 023237 (2020).

[34] C. Repellin and T. Senthil, Chern bands of twisted bilayer graphene: Fractional chern insulators and spin phase transition, Phys. Rev. Research 2, 023238 (2020).

[35] A. Abouelkomsan, Z. Liu, and E. J. Bergholtz, Particle-Hole Duality, Emergent Fermi Liquids, and Fractional Chern Insulators in Moiré Flatbands, Phys. Rev. Lett. 124, 106803 (2020).

[36] Z. Liu, A. Abouelkomsan, and E. J. Bergholtz, GateTunable Fractional Chern Insulators in Twisted Double Bilayer Graphene, Phys. Rev. Lett. 126, 026801 (2021).

[37] P. Wilhelm, T. C. Lang, and A. M. Läuchli, Interplay of Fractional Chern Insulator and Charge-Density-Wave Phases in Twisted Bilayer Graphene, Phys. Rev. B 103, 125406 (2021).

[38] R. Sohal, L. H. Santos, and E. Fradkin, Chern-simons composite fermion theory of fractional chern insulators, Phys. Rev. B 97, 125131 (2018).

[39] R. Sohal and E. Fradkin, Intertwined order in fractional chern insulators from finite-momentum pairing of composite fermions, Phys. Rev. B 101, 245154 (2020).

[40] Y. Xu, S. Liu, D. A. Rhodes, K. Watanabe, T. Taniguchi, J. Hone, V. Elser, K. F. Mak, and J. Shan, Correlated insulating states at fractional fillings of moiré superlattices, Nature (London) 587, 214 (2020).

[41] C. Jin, Z. Tao, T. Li, Y. Xu, Y. Tang, J. Zhu, S. Liu, K. Watanabe, T. Taniguchi, J. C. Hone, L. Fu, J. Shan, and K. F. Mak, Stripe phases in WSe2/WS2 moiré superlattices, Nat. Mater. 20, 940 (2021).

[42] Y. Zhou, J. Sung, E. Brutschea, I. Esterlis, Y. Wang, G. Scuri, R. J. Gelly, H. Heo, T. Taniguchi, K. Watanabe, G. Zaránd, M. D. Lukin, P. Kim, E. Demler, and H. Park, Signatures of bilayer Wigner crystals in a transition metal dichalcogenide heterostructure, arXiv:2010.03037.

[43] X. Huang, T. Wang, S. Miao, C. Wang, Z. Li, Z. Lian, T. Taniguchi, K. Watanabe, S. Okamoto, D. Xiao, S.-F. Shi, and Y.-T. Cui, Correlated insulating states at fractional fillings of the WS2/WSe2 moiré Lattice, arXiv:2007.11155.

[44] E. Tang, J.-W. Mei, and X.-G. Wen, High-Temperature Fractional Quantum Hall States, Phys. Rev. Lett. 106, 236802 (2011).

[45] K. Sun, Z. Gu, H. Katsura, and S. Das Sarma, Nearly Flatbands with Nontrivial Topology, Phys. Rev. Lett. 106, 236803 (2011).

[46] T. Neupert, L. Santos, C. Chamon, and C. Mudry, Fractional Quantum Hall States at Zero Magnetic Field, Phys. Rev. Lett. 106, 236804 (2011).

[47] N. Regnault and B. A. Bernevig, Fractional chern insulator, Phys. Rev. X 1, 021014 (2011).

[48] D. N. Sheng, Z.-C. Gu, K. Sun, and L. Sheng, Fractional quantum hall effect in the absence of landau levels, Nat. Commun. 2, 389 (2011).
[49] S. A. Parameswaran, R. Roy, and S. L. Sondhi, Fractional quantum Hall physics in topological flat bands, C. R. Phys. 14, 816 (2013)

[50] E. J. Bergholtz and Z. Liu, Topological flat band models and fractional chern insulators, Int. J. Mod. Phys. B 27, 1330017 (2013).

[51] Y.-L. Wu, B. A. Bernevig, and N. Regnault, Zoology of fractional chern insulators, Phys. Rev. B 85, 075116 (2012).

[52] F. Wu, T. Lovorn, E. Tutuc, and A. H. MacDonald, Hubbard Model Physics in Transition Metal Dichalcogenide Moiré Bands, Phys. Rev. Lett. 121, 026402 (2018).

[53] F. Wu, T. Lovorn, E. Tutuc, I. Martin, and A. H MacDonald, Topological Insulators in Twisted Transition Metal Dichalcogenide Homobilayers, Phys. Rev. Lett. 122, 086402 (2019).

[54] M. Levin and A. Stern, Fractional Topological Insulators, Phys. Rev. Lett. 103, 196803 (2009).

[55] D. Xiao, G.-B. Liu, W. Feng, X. Xu, and W. Yao, Coupled Spin and Valley Physics in Monolayers of $\operatorname{mos}_{2}$ and Other Group-Vi Dichalcogenides, Phys. Rev. Lett. 108, 196802 (2012).

[56] J. Y. Lee, E. Khalaf, S. Liu, X. Liu, Z. Hao, P. Kim, and A. Vishwanath, Theory of correlated insulating behaviour and spin-triplet superconductivity in twisted double bilayer graphene, Nat. Commun. 10, 5333 (2019).

[57] See Supplemental Materials at http://link.aps.org/supplemental/ 10.1103/PhysRevResearch.3.L032070 for (1) the variational calculations of the energy for the valley polarized and intervalley coherent state, (2) Hartree-Fock calculations, (3) a discussion on the possibility of the Halperin state, and (4) an effective theory for the transition between the valley polarized Fermi liquid and fractional Chern insulator.

[58] A. Stern, Fractional Topological Insulators: A Pedagogical Review, Annu. Rev. Condens. Matter Phys. 7, 349 (2016).

[59] One may think of another possibility, analogous to the Halperin $\left(m_{1}, m_{2}, n_{1}\right)$ states. However, this state is not favored because of the opposite Chern number in the opposite valley [57].

[60] B. A. Bernevig and N. Regnault, Emergent many-body translational symmetries of abelian and non-abelian fractionally filled topological insulators, Phys. Rev. B 85, 075128 (2012).

[61] S. L. Sondhi, A. Karlhede, S. A. Kivelson, and E. H. Rezayi, Skyrmions and the crossover from the integer to fractional quantum hall effect at small zeeman energies, Phys. Rev. B 47, 16419 (1993).

[62] S. Chatterjee, M. Ippoliti, and M. P. Zaletel, Skyrmion Superconductivity: DMRG evidence for a topological route to superconductivity, arXiv:2010.01144.

[63] K. Kumar, K. Sun, and E. Fradkin, Chern-simons theory of magnetization plateaus of the spin- $\frac{1}{2}$ quantum xxz heisenberg model on the kagome lattice, Phys. Rev. B 90, 174409 (2014).

[64] K. Kumar, K. Sun, and E. Fradkin, Chiral spin liquids on the kagome lattice, Phys. Rev. B 92, 094433 (2015).

[65] W. Zhu, S.-S. Gong, T.-S. Zeng, L. Fu, and D. N. Sheng, Interaction-Driven Spontaneous Quantum Hall Effect on a Kagome Lattice, Phys. Rev. Lett. 117, 096402 (2016). 